ESAIM: COCV 22 (2016) 404-416

DOI: $10.1051 / \mathrm{cocv} / 2015011$
ESAIM: Control, Optimisation and Calculus of Variations

www.esaim-cocv.org

\title{
AVERAGE-DISTANCE PROBLEM FOR PARAMETERIZED CURVES
}

\author{
Xin YANG LU ${ }^{1}$ AND DeJAN SlepČEV ${ }^{1}$
}

\begin{abstract}
We consider approximating a measure by a parameterized curve subject to length penalization. That is for a given finite compactly supported measure $\mu$, with $\mu\left(\mathbb{R}^{d}\right)>0$ for $p \geq 1$ and $\lambda>0$ we consider the functional

$$
E(\gamma)=\int_{\mathbb{R}^{d}} d\left(x, \Gamma_{\gamma}\right)^{p} \mathrm{~d} \mu(x)+\lambda \operatorname{Length}(\gamma)
$$

where $\gamma: I \rightarrow \mathbb{R}^{d}, I$ is an interval in $\mathbb{R}, \Gamma_{\gamma}=\gamma(I)$, and $d\left(x, \Gamma_{\gamma}\right)$ is the distance of $x$ to $\Gamma_{\gamma}$. The problem is closely related to the average-distance problem, where the admissible class are the connected sets of finite Hausdorff measure $\mathcal{H}^{1}$, and to (regularized) principal curves studied in statistics. We obtain regularity of minimizers in the form of estimates on the total curvature of the minimizers. We prove that for measures $\mu$ supported in two dimensions the minimizing curve is injective if $p \geq 2$ or if $\mu$ has bounded density. This establishes that the minimization over parameterized curves is equivalent to minimizing over embedded curves and thus confirms that the problem has a geometric interpretation.
\end{abstract}

Mathematics Subject Classification. 49Q20, 49K10, 49Q10, 35B65.

Received October 15, 2014. Revised February 8, 2015.

Published online March 8, 2016.

\section{INTRODUCTION}

Approximating measures by one dimensional objects arises in several fields. In the setting of optimization problems connected to network planning (such as for urban transportation network) and irrigation it was introduced by Buttazzo et al. [7], and has been extensively studied [2-6, 9, 16, 19, 22].

In this setting the problem is known as the average-distance problem. Given a set $\Sigma \subset \mathbb{R}^{d}$ let $d(x, \Sigma)=$ $\inf _{y \in \Sigma}|x-y|$. Let $\mathcal{M}$ be the set of finite, compactly supported measures in $\mathbb{R}^{d}$ for $d \geq 2$, with $\mu\left(\mathbb{R}^{d}\right)>0$.

Problem 1.1. Given a measure $\mu \in \mathcal{M}$, and parameters $p \geq 1, \lambda>0$, we consider the average-distance problem in the penalized (as opposed to constrained) form: minimize

$$
G_{\mu}^{\lambda, p}(\Sigma):=\int_{\mathbb{R}^{d}} d(x, \Sigma)^{p} \mathrm{~d} \mu(x)+\lambda \mathcal{H}^{1}(\Sigma),
$$

Keywords and phrases. Average-distance problem, principal curves, nonlocal variational problems.

1 Department of Mathematical Sciences, Carnegie Mellon University, Pittsburgh, PA, 15213, USA. xinyang@andrew.cmu.edu;

slepcev@math.cmu.edu 
with the unknown $\Sigma$ varying in the family

$$
\mathcal{A}:=\left\{\Sigma \subseteq \mathbb{R}^{d}: \Sigma \text { compact, path-wise connected }\right\} .
$$

Another application in which a measure is to be approximated by a one-dimensional object arises in machine learning and statistics where one wishes to obtain the curve that best represents the data given by a (probability) measure $\mu$. The problem in this setting was introduced by Hastie [12] and Hastie and Stuetzle [13], and its solution is known as the (regularized) principal curve. A variant of the problem can be formulated as follows: let

$$
\mathcal{C}:=\left\{\gamma:[0, a] \longrightarrow \mathbb{R}^{d}: a \geq 0, \gamma \text { is Lipschitz with }\left|\gamma^{\prime}\right| \leq 1 \text { a.e. }\right\} .
$$

For given $\gamma \in \mathcal{C}$, we define its length, $L(\gamma)$, as its total variation $\|\gamma\|_{T V([0, a])}$. Furthermore given $\gamma \in \mathcal{C}$ we denote its image by $\Gamma_{\gamma}=\gamma([0, a])$. The problem can be stated as follows:

Problem 1.2. Given a measure $\mu \in \mathcal{M}$, parameters $\lambda>0, p \geq 1$ find $\gamma \in \mathcal{C}$ minimizing

$$
E_{\mu}^{\lambda, p}(\gamma):=\int_{\mathbb{R}^{d}} d\left(x, \Gamma_{\gamma}\right)^{p} \mathrm{~d} \mu(x)+\lambda L(\gamma)
$$

The problem is different from the Problem 1.1 in that instead of minimizing over sets one minimizes over functions (parameterized curves). This makes the problem simpler in some aspects, in particular for numerical approximation of minimizers. However it is important to note that all competitors in Problem 1.1 are achievable as images of parameterized curves. From that perspective the main main difference between Problems 1.1 and 1.2 is in the way the length is penalized: as $\mathcal{H}^{1}$-measure of $\Gamma_{\gamma}$ in Problem 1.1, and as total variation of $\gamma$ in Problem 1.2. Thus if a curve $\gamma$ passes thrugh the same segment twice, then this length is counted twice by $\|\gamma\|_{T V}$, but only once by $\mathcal{H}^{1}\left(\Gamma_{\gamma}\right)$. We remark that in machine learning the problem has been considered most often with $p=2$, with a variety of regularizations, as well as with length constraint (instead of length penalization) $[15,24,25]$. Regularizing with a length term is the lowest order (in other words the weakest) of regularizations considered. We note that the first term of energy measures the approximation error while the second term penalizes the complexity of the approximating object (curve).

The existence of minimizers of Problem 1.2 in the class of parameterized curves follows from Ascoli-Arzelà theorem and the continuity of the functional with respect to the uniform convergence (Lem. 2.2). However it is not clear if for a general measure $\mu$ the minimizing curve is injective, in other words it may have self-intersections and not be an embedded curve. Here we show that in two dimensions if $p \geq 2$ then the minimizer in fact is an injective curve. We also show that if $\mu$ has bounded density with respect to the Lebesgue measure then the minimizer is an injective curve for all $1 \leq p<\infty$. More precisely the main result of our work is:

Theorem 1.3. Consider dimension $d=2$. Let $\mu \in \mathcal{M}$ and let $\lambda>0$ and $p \geq 1$. If $p<2$ assume that $\mu$ is absolutely continuous with respect to the Lebesgue measure and that its density, $\rho$, is bounded. Let $\gamma:[0, L] \rightarrow \mathbb{R}^{2}$ be an arc-length-parameterized minimizer of $E_{\mu}^{\lambda, p}$. Then $\gamma$ is injective and in particular $\Gamma_{\gamma}$ is a curve embedded in $\mathbb{R}^{2}$.

The theorem implies that the problem can also be posed as a minimization problem among embedded curves. We note that, as we discuss at the beginning of Section 4 , the conclusion of the theorem holds for all $1 \leq p<\infty$ if $\mu$ is a discrete measure.

We hypothesize that the range of $p$ in the theorem is sharp:

Conjecture 1.4. For $1 \leq p<2$ there exist $\lambda>0$ and a measure $\mu \in \mathcal{M}$ for which the global minimizer is not injective.

The proof of the Theorem relies on regularity properties of minimizers, which are of interest in their own right. In [23] it was shown that for $p=1$ minimizers of the Problem 1.1, even for measures with smooth densities, can be embedded curves which have a corner. Since these are also minimizers of Problem 1.2, we conclude that 
minimizers of Problem 1.2 are not $C^{1}$ curves in general. We remark that results of [23] can be extended to $p \geq 1$ to show existence of global minimizers which have a corner.

Consequently we consider regularity of minimizers in the sense of obtaining estimates on the total variation of $\gamma^{\prime}$, where $\gamma$ is an arc-length-parameterized minimizer. This allows us to consider the curvature as a measure and provides bounds on the total curvature of a segment of the minimizing curve in terms of the mass projecting on the segment. To do so we use techniques developed in [19].

This paper is structured as follows:

- In Section 2 we present preliminary notions and results, and prove existence of minimizers of Problem 1.2. We furthermore show that the minimizers are contained in the convex hull of the support of the measure $\mu$.

- In Section 3 we prove the injectivity of minimizers (Thm. 1.3) in the two-dimensional case.

- In Appendix we extend the regularity estimates of [19] to $p>1$ and prove them in the setting of parameterized curves. We furthermore provide the version of estimates in $\mathbb{R}^{2}$ which roughly speaking bounds how much a minimizer can turn to the left by the mass to the right of the curve. This is a key result needed to prove injectivity.

\section{Preliminaries}

In this section we provide some preliminary results including the proof of existence of minimizers of Problem 1.2 (Lem. 2.2).

We define the distance between curves in $\mathcal{C}$ as follows: Let $\gamma_{1}, \gamma_{2} \in \mathcal{C}$ with domains $\left[0, a_{1}\right],\left[0, a_{2}\right]$ respectively. We can assume that $a_{1} \leq a_{2}$. Let $\tilde{\gamma}_{1}:\left[0, a_{2}\right] \rightarrow \mathbb{R}^{d}$ be the extension of $\gamma_{1}$ to $\left[0, a_{2}\right]$ as follows

$$
\tilde{\gamma}_{1}(t)= \begin{cases}\gamma_{1}(t) & \text { if } t \in\left[0, a_{1}\right] \\ \gamma_{1}\left(a_{1}\right) & \text { if } t \in\left(a_{1}, a_{2}\right] .\end{cases}
$$

Let

$$
d_{\mathcal{C}}\left(\gamma_{1}, \gamma_{2}\right)=\max _{t \in\left[0, a_{2}\right]}\left|\tilde{\gamma}_{1}(t)-\gamma_{2}(t)\right| .
$$

The first issue is the existence of minimizers. A preliminary lemma is required. Given a measure $\mu \in \mathcal{M}$, and $p \geq 1$, let

$$
F_{\mu}^{p}: \mathcal{A} \longrightarrow[0, \infty), \quad F_{\mu}^{p}(\Sigma):=\int_{\mathbb{R}^{d}} d(x, \Sigma)^{p} \mathrm{~d} \mu(x) .
$$

Lemma 2.1. Given a measure $\mu \in \mathcal{M}$, parameters $\lambda>0, p \geq 1$, then for any minimizing sequence $\left\{\gamma_{n}\right\}$ of Problem 1.2 it holds:

- length estimate:

$$
\limsup _{n \rightarrow \infty} L\left(\gamma_{n}\right) \leq \frac{\mu\left(\mathbb{R}^{d}\right)}{\lambda}(\operatorname{diam} \operatorname{supp}(\mu))^{p},
$$

- confinement condition: there exists a compact set $K \subseteq \mathbb{R}^{d}$ such that $\Gamma_{\gamma_{n}} \subseteq K$ for all $n$.

Proof. Boundedness of the length is obtained by using a singleton as a competitor. Fix an arbitrary point $z \in \operatorname{supp}(\mu)$, and let $\gamma:[0,0] \longrightarrow\{z\}$. Then

$$
\inf _{\mathcal{C}} E_{\mu}^{\lambda, p} \leq E_{\mu}^{\lambda, p}(\gamma) \leq \int_{\mathbb{R}^{d}}|x-z|^{p} \mathrm{~d} \mu(x) \leq \mu\left(\mathbb{R}^{d}\right)(\operatorname{diam} \operatorname{supp}(\mu))^{p} .
$$

Since $\left\{\gamma_{n}\right\}$ is a minimizing sequence, (2.2) gives

$$
(\forall \varepsilon)\left(\exists n_{0}\right)\left(\forall n \geq n_{0}\right) \quad \lambda L\left(\gamma_{n}\right) \leq E_{\mu}^{\lambda, p}\left(\gamma_{n}\right) \leq \mu\left(\mathbb{R}^{d}\right)(\operatorname{diam} \operatorname{supp}(\mu))^{p}+\varepsilon .
$$


To prove the confinement condition, note that for any $r \geq 0, \gamma \in \mathcal{C}$ it holds

$$
\Gamma_{\gamma} \cap(\operatorname{supp}(\mu))_{r}=\emptyset \Longrightarrow E_{\mu}^{\lambda, p}(\gamma) \geq F_{\mu}^{p}\left(\Gamma_{\gamma}\right) \geq \mu\left(\mathbb{R}^{d}\right) r^{p}
$$

where $(\operatorname{supp}(\mu))_{r}=\left\{x \in \mathbb{R}^{d}: \inf _{y \in \operatorname{supp}(\mu)}|x-y| \leq r\right\}$. Thus (2.2) gives

$$
(\forall \varepsilon)\left(\exists n_{0}\right): \quad\left(\forall n \geq n_{0}\right) \quad F_{\mu}^{p}\left(\Gamma_{\gamma}\right) \leq E_{\mu}^{\lambda, p}\left(\gamma_{n}\right) \leq \mu\left(\mathbb{R}^{d}\right)(\operatorname{diam} \operatorname{supp}(\mu))^{p}+\varepsilon,
$$

and combining with length estimate $(2.3)$ and taking $\varepsilon=1$ gives

$$
\left(\exists n_{0}\right)\left(\forall n \geq n_{0}\right) \quad \Gamma_{\gamma_{n}} \subseteq(\operatorname{supp}(\mu))_{\left(\operatorname{diam} \operatorname{supp}(\mu)+\mu\left(\mathbb{R}^{d}\right)(\operatorname{diam} \operatorname{supp}(\mu))^{p} / \lambda+1+1 / \lambda\right)}
$$

concluding the proof.

Given a measure $\mu \in \mathcal{M}$ and a curve $\gamma$, let $\pi$ be a finite measure supported on $\mathbb{R}^{d} \times \Gamma_{\gamma}$ such that the first marginal of $\pi$ is $\mu$ and that for $\pi$-a.e. $(x, y),|x-y|=\min _{z \in \Gamma_{\gamma}}|x-z|$. The existence of such a measure is proved in (Lem. 2.1 of [19]). Let $\sigma$ be the second marginal of $\pi$. Then $\sigma$ is supported on $\Gamma_{\gamma}$ and $\pi$ is an optimal transportation plan between $\mu$ and $\sigma$ for the $\operatorname{cost} c(x, y)=|x-y|^{q}$, for any $q \geq 1$. In other words $\sigma$ is a projection of $\mu$ onto $\Gamma_{\gamma}$.

We remark that in $[20]$ it has been prove d that for any $\Sigma \in \mathcal{A}$, the ridge

$$
\mathfrak{R}_{\Sigma}:=\{x: \text { there exist } p, q \in \Sigma, p \neq q,|x-p|=|x-q|=d(x, \Sigma)\}
$$

is $\mathcal{H}^{1}$-rectifiable. Thus for any $\Sigma \in \mathcal{A}$ the (point-valued) "projection" map

$$
\Pi_{\Sigma}: \mathbb{R}^{d} \longrightarrow \Sigma, \quad \Pi_{\Sigma}(x):=\text { the point of } \Sigma \text { such that }|x-\pi(x)|=d(x, \Sigma)
$$

is well defined $\mathcal{L}^{2}$-a.e. Consequently if $\mu$ is absolutely continuous with respect to Lebesgue measure the measures $\pi$ and $\sigma$ above are uniquely defined and furthermore $\sigma=\Pi_{\Gamma_{\gamma} \sharp \mu}$.

Lemma 2.2. Consider a positive measure $\mu$ and parameters $\lambda>0, p \geq 1$. Problem 1.2 has a minimizer $\gamma \in \mathcal{C}$. Furthermore, for any minimizer, $\Gamma_{\gamma}$ is contained in $\operatorname{Conv}(\mu)$, the convex hull of the support of $\mu$.

A version of the second part of the statement has been proved in Proposition 5.1 of [3]. For completeness, we provide a simple proof here.

We note that since the energy is invariant under reparameterizing the curve it follows that the problem has a minimizer $\gamma \in \mathcal{C}$ which is arc-length parameterized.

Proof. Consider a minimizing sequence $\left\{\gamma_{n}\right\}$ in $\mathcal{C}$. Since a reparameterization does not change the value of the functional we can assume that $\gamma_{n}$ are arc-length parameterized for all $n$. Lemma 2.1 proves that $\left\{\gamma_{n}\right\}$ are uniformly bounded and have uniformly bounded lengths. Let $L$ be the supremum of the lengths and let $\tilde{\gamma}_{n}$ be the extensions of the curves as in $(2.1)$ to interval $[0, L]$. The curves $\left\{\tilde{\gamma}_{n}\right\}$ satisfy the conditions of Arzelà-Ascoli Theorem. Thus, along a subsequence (which we assume to be the whole sequence) they converge uniformly (and thus in $\mathcal{C}$ ) to a curve $\gamma:[0, L] \rightarrow \mathbb{R}^{d}$. Since all of the curves are 1-Lipschitz, so is $\gamma$ and thus it belongs to $\mathcal{C}$.

Since $\varphi \mapsto F_{\mu}^{p}\left(\Gamma_{\varphi}\right)$ is continuous and $\varphi \mapsto L(\varphi)$ is lower-semicontinuous with respect to the convergence in $\mathcal{C}$, it follows $\liminf \operatorname{in}_{n \rightarrow \infty} E_{\mu}^{\lambda, p}\left(\tilde{\gamma}_{n}\right) \geq E_{\mu}^{\lambda, p}(\gamma)$. Since $\left\{\tilde{\gamma}_{n}\right\}_{n}$ is also a minimizing sequence, $\gamma$ is a minimizer of $E_{\mu}^{\lambda, p}$.

Now we prove that any minimizer is contained in the convex hull of $\operatorname{supp}(\mu)$. The argument relies on fact that the projection onto a convex set decreases length, which follows from the 1-Lipschitz continuity of projection maps, as proven in Proposition 5.3 in the book by Brezis [1]. Let $\gamma \in \mathcal{C}$ be a minimizer of $E_{\mu}^{\lambda, p}$. Assume it is not contained in the convex hull, $K=\operatorname{Conv}(\mu)$, of the support of $\mu$. Then there exists $T \in[0, a]$ such that $\gamma(T) \notin K$. Let $\left[t_{1}, t_{2}\right]$ be the maximal interval such that $\gamma\left(\left(t_{1}, t_{2}\right)\right) \cap K=\emptyset$. We claim that $\sigma\left(\gamma\left(t_{1}, t_{2}\right)\right)=0$. Otherwise consider $\tilde{\gamma}$ be the projection of $\gamma$ onto $K$. The distances between $\tilde{\gamma}(t)$ and points in $K$ are strictly 


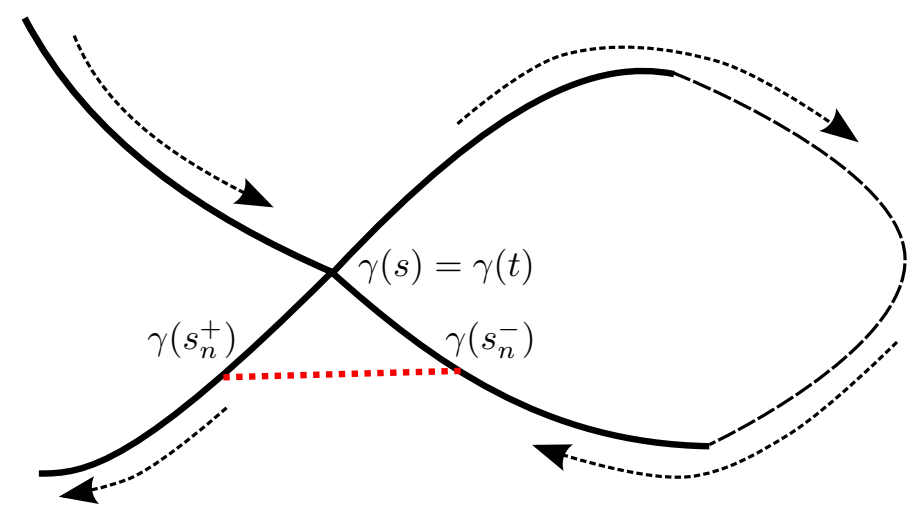

Figure 1. This is a schematic representation of the variation. The black lines belong to the (graph of) $\gamma$, while the red dotted line belongs to the (graph of) competitor $\tilde{\gamma}_{n}$. Time increases along the direction of the arrows.

less than the distances between $\gamma(t)$ and the points in $K$ and thus $F_{\mu}^{p}\left(\Gamma_{\tilde{\gamma}}\right)<F_{\mu}^{p}\left(\Gamma_{\gamma}\right)$. Since the projection map is 1-Lipschitz, the length of $\tilde{\gamma}$ is less than or equal to the length of $\gamma$. Consequently $E_{\mu}^{\lambda, p}(\tilde{\gamma})<E_{\mu}^{\lambda, p}(\gamma)$, which contradicts the assumption that $\gamma$ is a minimizer. Thus $\sigma\left(\gamma\left(t_{1}, t_{2}\right)\right)=0$.

If $\gamma\left(t_{1}\right)$ and $\gamma\left(t_{2}\right)$ belong to $K$ then consider $\gamma_{2}$ obtained by replacing the segment $\left.\gamma\right|_{\left[t_{1}, t_{2}\right]}$ of $\gamma$ by a straight line segment. Note that the length of $\gamma_{2}$ is less than the length of $\gamma$ (since otherwise $\left.\gamma\right|_{\left(t_{1}, t_{2}\right)}$ would have to be a line segment which contradicts the fact that it is outside of $K)$. Also note that $F_{\mu}^{p}\left(\Gamma_{\gamma_{2}}\right)=F_{\mu}^{p}\left(\Gamma_{\gamma}\right)$ and thus $E_{\mu}^{\lambda, p}\left(\gamma_{2}\right)<E_{\mu}^{\lambda, p}(\gamma)$, which contradicts the assumption that $\gamma$ is a minimizer.

If $\gamma\left(t_{1}\right) \notin K$ then $t_{1}=0$. Noting that $\gamma_{2}=\left.\gamma\right|_{\left[t_{2}, a\right]}$ has lower energy than $\gamma$ contradicts the minimality of $\gamma$. The case $\gamma\left(t_{2}\right) \notin K$ is analogous.

\section{INJECTIVITY}

The main aim of this section is to prove injectivity for minimizers of Problem 1.2 in two dimensions. We say that $P \in \Gamma_{\gamma}$ is a double point if $\gamma^{-1}(P)$ has at least two elements. Our goal is to show that there are no double points. Note that if $\Gamma_{\gamma}$ is a simple curve, then it admits an injective parameterization, which is shorter than any noninjective parameterization. Thus to show that noninjectivity leads to contradiction one only needs to consider minimizers where $\Gamma_{\gamma}$ contains a point of order 3, that is points $P$ such that for $r>0$ small enough $\left(\Gamma_{\gamma} \cap B(P, r)\right) \backslash\{P\}$ has at least three connected components. We do not use this fact explicitly, but we do basically focus on a branching point, whose existence is established in Lemma 3.2.

Lemma 3.1. Let $\mu \in \mathcal{M}$ and let $\lambda>0$ and $p \geq 1$. Let $\gamma:[0, L] \rightarrow \mathbb{R}^{d}$ be an arc-length-parameterized minimizer of $E_{\mu}^{\lambda, p}$. Assume there exist times $0<t<s<L$ such that $\gamma(t)=\gamma(s)$. Then $\gamma$ is differentiable at $t$ and at $s$.

Furthermore $\gamma^{\prime}(t)=\gamma^{\prime}(s)$ or $\gamma^{\prime}(t)=-\gamma^{\prime}(s)$.

Proof. Assume the claim does not hold. Without a loss of generality we can assume that $\gamma$ is not differentiable at $s$. By Remark A.3 the one sided derivatives $\gamma^{\prime}(s-)$ and $\gamma^{\prime}(s+)$ exist and have unit lengths. Since $\gamma$ is not differentiable at $s, \gamma^{\prime}(s-) \neq \gamma^{\prime}(s+)$. Given that $\gamma^{\prime}(s-)$ and $\gamma^{\prime}(s+)$ are unit vectors it follows that they are not positive multiples of each other.

Consequently there exist sequences $\left\{s_{n}^{-}\right\} \searrow s,\left\{s_{n}^{+}\right\} \nearrow s$, such that $\angle \gamma\left(s_{n}^{-}\right) \gamma(s) \gamma\left(s_{n}^{+}\right) \rightarrow \alpha<\pi$ as $n \rightarrow \infty$. Note that by Lemma 2.1 for all $z \in \operatorname{supp}(\mu)$ and all $y \in \Gamma_{\gamma},|z-y|<\operatorname{diam}(\operatorname{supp}(\mu))$. 
Consider the competitors $\tilde{\gamma}_{n}$ constructed in the following way: let

$$
\begin{array}{ll}
\xi_{n}^{*}:[0,1] \longrightarrow \mathbb{R}^{d}, & \xi_{n}^{*}(u):=(1-u) \gamma\left(s_{n}^{-}\right)+u \gamma\left(s_{n}^{+}\right), \\
\xi_{n}:\left[0,\left|\gamma\left(s_{n}^{-}\right)-\gamma\left(s_{n}^{+}\right)\right|\right] \longrightarrow \mathbb{R}^{d}, & \xi_{n}(u):=\xi_{n}^{*}\left(u /\left|\gamma\left(s_{n}^{-}\right)-\gamma\left(s_{n}^{+}\right)\right|\right) .
\end{array}
$$

Let $\tilde{\gamma}_{n}:\left[0, L(\gamma)-\left(s_{n}^{+}-s_{n}^{-}\right)+\left|\gamma\left(s_{n}^{-}\right)-\gamma\left(s_{n}^{+}\right)\right|\right]$, where

$$
\tilde{\gamma}_{n}(u):=\left\{\begin{array}{cl}
\gamma(u) & \text { if } u \leq s_{n}^{-}, \\
\xi_{n}\left(u-s_{n}^{-}\right) & \text {if } s_{n}^{-} \leq u \leq s_{n}^{-}+\left|\gamma\left(s_{n}^{-}\right)-\gamma\left(s_{n}^{+}\right)\right|, \\
\gamma\left(u-s_{n}^{-}-\left|\gamma\left(s_{n}^{-}\right)-\gamma\left(s_{n}^{+}\right)\right|+s_{n}^{+}\right) & \text {if } u \geq s_{n}^{-}+\left|\gamma\left(s_{n}^{-}\right)-\gamma\left(s_{n}^{+}\right)\right| .
\end{array}\right.
$$

Since by hypothesis $\angle \gamma\left(s_{n}^{-}\right) \gamma(s) \gamma\left(s_{n}^{+}\right) \rightarrow \alpha \neq 0$, it follows (for any sufficiently large $n$ )

$$
\left|s_{n}^{+}-s_{n}^{-}\right|-\left|\gamma\left(s_{n}^{-}\right)-\gamma\left(s_{n}^{+}\right)\right| \geq c\left|s_{n}^{+}-s_{n}^{-}\right|,
$$

for some constant $c>0$ independent of $n$. Hence

$$
L(\gamma) \geq L\left(\tilde{\gamma}_{n}\right)+c\left|s_{n}^{+}-s_{n}^{-}\right| .
$$

By taking $n$ large we can assume that $\left|s_{n}^{+}-s_{n}^{-}\right|<1$.

We claim that

$$
F_{\mu}^{p}\left(\tilde{\gamma}_{n}\right)-F_{\mu}^{p}(\gamma) \leq \mu\left(\left\{z: \operatorname{argmin}_{y \in \Gamma_{\gamma}}|z-y| \subseteq \gamma\left(\left(s_{n}^{-}, s_{n}^{+}\right) \backslash\{s\}\right)\right\}\right) p D^{p-1}\left|s_{n}^{+}-s_{n}^{-}\right|,
$$

where $D:=1+\operatorname{diam} \operatorname{supp}(\mu)$. Note that if a point $z$ satisfies

$$
d\left(z, \Gamma_{\gamma}\right)<d\left(z, \Gamma_{\tilde{\gamma}_{n}}\right)
$$

then $\operatorname{argmin}_{y \in \Gamma_{\gamma}}|z-y| \subseteq \gamma\left(\left(s_{n}^{-}, s_{n}^{+}\right) \backslash\{s\}\right)$.

The constant $p D^{p-1}$ is due to the fact that any such point $z \in \operatorname{supp}(\mu)$ satisfies, due to Lemma 2.2 and construction of $\tilde{\gamma}_{n}$,

$$
\max \left\{d\left(z, \Gamma_{\gamma}\right), d\left(z, \Gamma_{\tilde{\gamma}_{n}}\right)\right\} \leq D .
$$

By construction there exists a point $z_{n}^{\prime} \in \Gamma_{\tilde{\gamma}_{n}} \backslash \Gamma_{\gamma}$ satisfying $\left|z-z_{n}^{\prime}\right|=d\left(z, \Gamma_{\tilde{\gamma}_{n}}\right)$. Denoting by $z^{\prime} \in \gamma\left(\left(s_{n}^{-}, s_{n}^{+}\right) \backslash\{s\}\right)$ a point satisfying $\left|z-z^{\prime}\right|=d\left(z, \Gamma_{\gamma}\right)$, we conclude

$$
|| z-\left.z^{\prime}\right|^{p}-\left|z-z_{n}^{\prime}\right|^{p}\left|\leq p D^{p-1}\right| s_{n}^{-}-s_{n}^{+} \mid .
$$

Since $\bigcap_{n} \gamma\left(\left(s_{n}^{-}, s_{n}^{+}\right) \backslash\{s\}\right)=\emptyset$, it follows that

$$
\lim _{n \rightarrow \infty} \mu\left(\left\{z: \operatorname{argmin}_{y \in \Gamma_{\gamma}}|z-y| \subseteq \gamma\left(\left(s_{n}^{-}, s_{n}^{+}\right) \backslash\{s\}\right)\right\}\right)=0 .
$$

Combining with (3.1) gives that the minimality of $\gamma$ is contradicted by $\tilde{\gamma}_{n}$ for sufficiently large $n$.

To show the second claim assume that $\gamma^{\prime}(t) \neq \gamma^{\prime}(s)$ and $\gamma^{\prime}(t) \neq-\gamma^{\prime}(s)$. Consider the following "reparameterization" of the curve $\gamma$. Let $\tilde{\gamma}:[0, L] \rightarrow \mathbb{R}^{d}$ be defined by

$$
\tilde{\gamma}(r)= \begin{cases}\gamma(r) & \text { for } r \in[0, t] \\ \gamma(s-(r-t)) & \text { for } r \in(t, s] \\ \gamma(r) & \text { for } r \in(s, L]\end{cases}
$$

Then $\tilde{\gamma}$ is also a minimizer of $E_{\mu}^{\lambda, p}$. However $\tilde{\gamma}$ is not differentiable at $t$ (and at $s$ ), which contradicts the first part of the lemma. 
Lemma 3.2. Let $\mu \in \mathcal{M}$ and let $\lambda>0$ and $p \geq 1$. Let $\gamma:[0, L] \rightarrow \mathbb{R}^{d}$ be an arc-length-parameterized minimizer of $E_{\mu}^{\lambda, p}$. There exists $\delta_{1} \in(0,1)$ such that $\gamma$ is injective on $\left[0, \delta_{1}\right)$ and $\left(L-\delta_{1}, L\right]$. Moreover the set of double points

$$
\mathcal{D}:=\{t \in[0, L]: \gamma(t) \text { is a double point }\},
$$

contais its infimum and supremum, that is $\inf \mathcal{D} \in \mathcal{D}$ and $\sup \mathcal{D} \in \mathcal{D}$.

Proof. Assume there exist no $\delta_{2}$ such that $\gamma$ is injective on $\left[0, \delta_{2}\right)$. Then there exist sequences $r_{k} \rightarrow 0$ and $s_{k}$ such that $r_{k}<s_{k}$ and $\gamma\left(r_{k}\right)=\gamma\left(s_{k}\right)$. Three cases arise.

- If $\gamma(0)$ is a double point, i.e. there exists $s \neq 0$ with $\gamma(s)=\gamma(0)$, then for sufficiently small $\delta_{2}$ the competitor $\gamma_{\mid\left[\delta_{2}, L\right]}$ contradicts the minimality of $\gamma$. This follows by a direct check, using that the mass projected of $\gamma\left(\left(0, \delta_{2}\right)\right)$ decreases to zero as $\delta_{2} \rightarrow 0$.

- If $\inf _{k} s_{k}=0$, then by considering subsequences we can assume that $\lim _{k \rightarrow \infty} s_{k}=0$, and $r_{k}<s_{k}<$ $r_{k-1}$ for all $k$. Then the intervals $\left[r_{k}, s_{k}\right]$ are all mutually disjoint. Since $\gamma\left(s_{k}\right)=\gamma\left(r_{k}\right)$ we conclude that $\left\|\gamma^{\prime}\right\|_{T V\left(r_{k}, s_{k}\right)} \geq \pi$ which implies that $\left\|\gamma^{\prime}\right\|_{T V([0, L])}$ is infinite. This contradicts the regularity estimate of Proposition A.2.

- If $\inf _{k} s_{k}>0$, then by considering a subsequence we can assume that $\lim _{k \rightarrow \infty} s_{k}=s$ for some $s \in(0, L]$. The continuity of $\gamma$ gives that $\gamma(0)=\gamma(s)$, which reduces the argument to the first case.

Thus all three cases lead to a contradiction. Proof of injectivity on $\left(L-\delta_{1}, L\right]$ is analogous.

To prove $t_{0}:=\inf \mathcal{D} \in \mathcal{D}$, consider a sequence $t_{k}$ in $\mathcal{D}$ such that $t_{k} \searrow t_{0}$. Since $\gamma\left(t_{k}\right)$ are all double points, there exists $t_{k}^{\prime} \neq t_{k}$ such that $\gamma\left(t_{k}\right)=\gamma\left(t_{k}^{\prime}\right)$. By considering subsequences we assume $t_{k}^{\prime} \rightarrow t_{0}^{\prime}$. The continuity of $\gamma$ gives $\gamma\left(t_{k}^{\prime}\right) \rightarrow \gamma\left(t_{0}^{\prime}\right)$. If $t_{0}^{\prime} \neq t_{0}$, it follows $t_{0} \in \mathcal{D}$. If $t_{0}^{\prime}=t_{0}$, and hence $t_{k}^{\prime} \searrow t_{0}$, by considering subsequences we can assume that $t_{k}^{\prime}<t_{k}<t_{k-1}^{\prime}$ Then the intervals $\left[t_{k}^{\prime}, t_{k}\right]$ are all mutually disjoint. Since $\gamma\left(t_{k}\right)=\gamma\left(t_{k}^{\prime}\right)$ we conclude that $\left\|\gamma^{\prime}\right\|_{T V\left(t_{k}^{\prime}, t_{k}\right)} \geq \pi$ which implies that $\left\|\gamma^{\prime}\right\|_{T V([0, L])}$ is infinite. This contradicts the regularity estimate of Proposition A.2. The same arguments applied to $\tilde{\gamma}:[0, L] \rightarrow \mathbb{R}^{d}, \tilde{\gamma}(s):=\gamma(L-s)$, prove $\sup \mathcal{D} \in \mathcal{D}$.

Proof of Theorem 1.3. Let $\gamma:[0, L] \rightarrow \mathbb{R}^{2}$ be an arc-length-parameterized minimizer of $E_{\mu}^{\lambda, p}$, and let $\Gamma_{\gamma}=$ $\gamma([0, L])$. Recall that $P \in \Gamma_{\gamma}$ is a double point if $\gamma^{-1}(P)$ has at least two elements. Our goal is to show that $\Gamma_{\gamma}$ has no double points.

By Lemma 3.2 there exists $\delta_{1}>0$ such that $\gamma\left(\left[0, \delta_{1}\right)\right)$ and $\gamma\left(\left(L-\delta_{1}, L\right]\right)$ contain no double points. Assume that there are double points on $\gamma\left(\left[\delta_{1}, L-\delta_{1}\right]\right)$. Let

$$
t_{2}=\sup \left\{t<L-\delta_{1}: \gamma(t) \text { is a double point }\right\} .
$$

Note that $\gamma$ is injective on $\left(t_{2}, L\right]$. By Lemma $3.2, \gamma\left(t_{2}\right)$ is a double point. Hence, for sufficiently small $\delta_{1}$, there exists $t_{1} \in\left(\delta_{1}, t_{2}\right)$ such that $\gamma\left(t_{1}\right)=\gamma\left(t_{2}\right)$. By Lemma 3.1, there are two possibilities: either $\gamma^{\prime}\left(t_{1}\right)=\gamma^{\prime}\left(t_{2}\right)$ or $\gamma^{\prime}\left(t_{1}\right)=-\gamma^{\prime}\left(t_{2}\right)$. Since the arguments are analogous we assume $\gamma^{\prime}\left(t_{1}\right)=\gamma^{\prime}\left(t_{2}\right)$. By regularity of $\gamma$ established in (A.11), there exists $\delta_{2} \in\left(0, \delta_{1}\right)$ such that $\left\|\gamma^{\prime}\right\|_{T V\left(t_{1}, t_{1}+\delta_{2}\right)}<\frac{1}{8}$ and $\left\|\gamma^{\prime}\right\|_{T V\left(t_{2}, t_{2}+\delta_{2}\right)}<\frac{1}{8}$. Therefore $\gamma$ restricted to $\left[t_{1}, t_{1}+\delta_{2}\right]$ is injective. Since $\gamma\left(\left(t_{2}, t_{2}+\delta_{2}\right)\right)$ has no double points $\gamma\left(\left(t_{1}, t_{1}+\delta_{2}\right)\right) \cap \gamma\left(\left(t_{2}, t_{2}+\delta_{2}\right)\right)=\emptyset$.

We can assume without a loss of generality that $\gamma\left(t_{1}\right)=0$ and $\gamma^{\prime}\left(t_{1}\right)=e_{1}$. The bound on total variation of $\gamma^{\prime}$ above implies that $\gamma^{\prime} \cdot e_{1}>\frac{7}{8}$ on the intervals considered. Therefore we can reparameterize the curve using the first coordinate as the parameter. That is there exists Lipschitz functions $x, \alpha, \beta:\left[0, \frac{7}{8} \delta_{2}\right) \rightarrow \mathbb{R}$ such that $\frac{7}{8}<x^{\prime}(s) \leq 1$ a.e. and for all $s \in\left[0, \delta_{2}\right]$,

$$
\gamma_{1}\left(t_{1}+s\right)=(x(s), \alpha(x(s))) \quad \text { and } \quad \gamma_{2}\left(t_{2}+s\right)=(x(s), \beta(x(s)))
$$

define two branches of $\gamma$. Let $\delta=\delta_{2} / 3$. Without a loss of generality we can assume that $\alpha>\beta$ on $(0, \delta)$.

Our goal is to arrive at contradiction by showing that $\alpha=\beta$ on some interval $[0, \tilde{\delta})$. The reason is that $\alpha$ cannot separate from $\beta$ is that for $\alpha$ to turn upward, by Lemma A.4, there must be mass beneath $\alpha$ talking to 


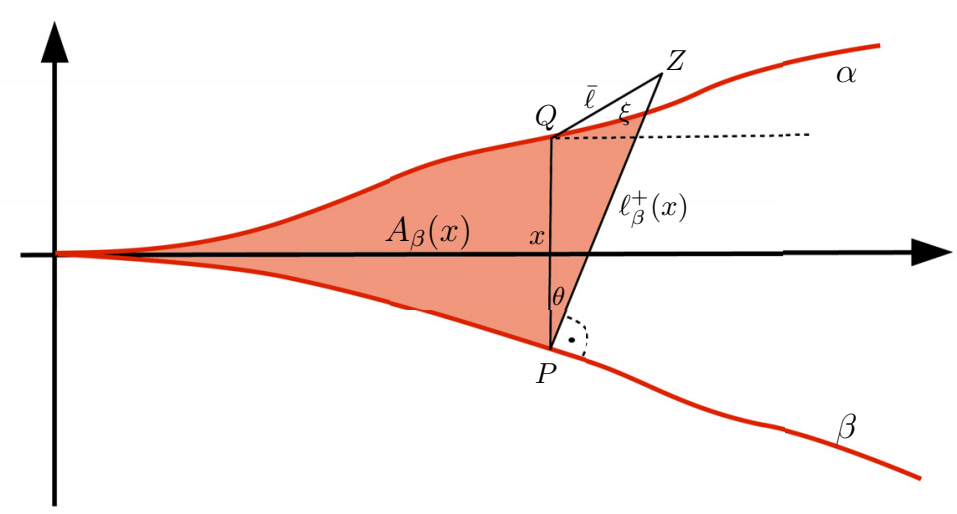

FiguRE 2. The geometry of the configuration near the last double point.

that part of the curve. But the mass beneath $\alpha$ which talks to $\alpha$ must lie above $\beta$. However the region between $\alpha$ and $\beta$ cannot contain enough mass to allow for the needed turn. Below we make this argument precise. For a.e. $x \in[0, \delta), \alpha$ and $\beta$ are differentiable at $x$ and we define $\ell_{\alpha}^{+}(x)=\left\{(x, \alpha(x))+r\left(\alpha^{\prime}(x), 1\right): r \geq 0\right\}$ to be the halfline perpendicular to $\alpha$ at $x$ extending above $\alpha$ and $\ell_{\alpha}^{-}(x)=\left\{(x, \alpha(x))+r\left(\alpha^{\prime}(x), 1\right): r \leq 0\right\}$ the halfline below. The halflines $\ell_{\beta}^{+}(x)$ and $\ell_{\beta}^{-}(x)$ are defined analogously, as illustrated in Figure 2.

Let $S_{\alpha}(x)=\{(z, \alpha(z)): z \in[0, x]\}$ and $S_{\beta}(x)=\{(z, \beta(z)): z \in[0, x]\}$. Let $U_{\alpha}(x)$ be the connected component of $\mathbb{R}^{2} \backslash\left(S_{\alpha}(x) \cup \ell_{\alpha}^{-}(x) \cup S_{\beta}(\delta)\right)$ containing the point $(x / 2,(\alpha(x / 2)+\beta(x / 2)) / 2)$. Analogously we define $A_{\beta}(x)$ be the connected component of $\mathbb{R}^{2} \backslash\left(S_{\alpha}(\delta) \cup \ell_{\beta}^{+}(x) \cup S_{\beta}(x)\right)$ containing the point $(x / 2,(\alpha(x / 2)+\beta(x / 2)) / 2)$. Note that all of the mass below the curve $\alpha$ and talking to $S_{\alpha}(x)$ is a subset of $U_{\alpha}(x)$. Likewise all of the mass above the curve $\beta$ and talking to $S_{\beta}(x)$ is a subset of $A_{\beta}(x)$.

We introduce:

$$
f(x)=\sup _{0 \leq z \leq x} \frac{\alpha^{\prime}(z)}{\left|\left(1, \alpha^{\prime}(z)\right)\right|} \quad \text { and } \quad g(x)=\inf _{0 \leq z \leq x} \frac{\beta^{\prime}(z)}{\left|\left(1, \beta^{\prime}(z)\right)\right|} .
$$

Note that $f(x)-g(x)>0$ on $(0, \delta)$ and that, using the assumption on total variation of $\gamma^{\prime}$, it follows that for $x \in[0, \delta),|f(x)|$ and $|g(x)|$ are less than $\frac{1}{8}$.

Let $\bar{\ell}$ be the line passing through $Q=(x, \alpha(x))$ whose slope is $\frac{1}{8}$. It stays above the graph of $\alpha$ on $(x, \delta)$. Let $Z$ be the intersection point of $\bar{\ell}$ and $\ell_{\beta}^{+}(x)$. We note that $\theta:=\angle Z P Q<\arctan \left(\frac{1}{8}\right)$ and thus $[Q Z]$ is the shortest side of the triangle $\triangle P Z Q$. Therefore

$$
|P Z|<2|Q P|=2(\alpha(x)-\beta(x)) \leq 2 x(f(x)-g(x)) .
$$

Since $f(x)-g(x)$ is a nondecreasing function we conclude that

$$
\sup _{z \in A_{\beta}(x)} d\left(z, \Gamma_{\gamma}\right) \leq 2 x(f(x)-g(x))
$$

Likewise

$$
\sup _{z \in U_{\alpha}(x)} d\left(z, \Gamma_{\gamma}\right) \leq 2 x(f(x)-g(x)) .
$$

Lemma A.4 implies that

$$
f(x) \leq \frac{p}{\lambda}(2 x(f(x)-g(x)))^{p-1} \mu\left(U_{\alpha}(x)\right) \quad \text { and } \quad g(x) \geq-\frac{p}{\lambda}(2 x(f(x)-g(x)))^{p-1} \mu\left(A_{\beta}(x)\right) .
$$

We first focus on $p \geq 2$. From the above inequalities it follows that for some constant $c>0$

$$
(f(x)-g(x))^{2-p} \leq c x^{p-1} .
$$


Since as $x \rightarrow 0^{+}$the left-hand side has uniform positive lower bound while the right-hand side converges to zero we obtain a contradiction, as desired.

We now consider the more delicate case: $1 \leq p<2$. Recall that we now assume that $\mu$ has bounded density $\rho$. To obtain a bound on $\mu\left(A_{\beta}(x)\right)$ we estimate the area of $A_{\beta}(x)$. The area of $A_{\beta}(x)$ is bounded from above by the sum of the areas of the region between the curves to the left of line segment $[P Q]$ and the area of triangle $P Z Q$ in Figure 2.

We note that $\theta<\arctan \left(\frac{1}{8}\right)$ and the angle $\angle Z Q P$ is $\frac{\pi}{2}+\arctan \left(\frac{1}{8}\right)$. Therefore $\xi=\angle Q Z P>\frac{\pi}{6}$. Using the law of sines and $\alpha(x)-\beta(x) \leq x(f(x)-g(x))$ we obtain

$$
\frac{1}{2 x(f(x)-g(x))}<\frac{\sin \xi}{\alpha(x)-\beta(x)}=\frac{\sin \theta}{|Q Z|}<\frac{1}{8|Q Z|} .
$$

Therefore

$$
\operatorname{Area}(\triangle P Z Q) \leq \frac{1}{2}|Q P| \cdot|Q Z| \leq \frac{1}{8} x^{2}(f(x)-g(x))^{2}
$$

Consequently

$$
\text { Area }\left(A_{\beta}(x)\right) \leq x^{2}(f(x)-g(x))+x^{2}(f(x)-g(x))^{2} \leq 2 x^{2}(f(x)-g(x)) .
$$

Same upper bound holds for $\operatorname{Area}\left(U_{\alpha}(x)\right)$. Therefore

$$
\max \left\{\mu\left(U_{\alpha}(x)\right), \mu\left(A_{\beta}(x)\right)\right\} \leq 2\|\rho\|_{L^{\infty}} x^{2}(f(x)-g(x)) .
$$

Combining with estimate (3.4), (choosing $\delta_{2}$ sufficiently small) and using that $2 x(f(x)-g(x))<1$ gives that for a.e $x \in[0, \delta)$

$$
0 \leq f(x)-g(x) \leq 4 \frac{p}{\lambda}\|\rho\|_{L^{\infty}} x^{2}(f(x)-g(x)) .
$$

This implies that for a.e. $x>0$ small enough $f(x)=0$ and $g(x)=0$, which means that the curves coincide. Contradiction.

We remark that the statement of Theorem 1.3 can be extended to measures $\mu \ll \mathcal{L}^{2}$ with Radon-Nikodym derivative $\rho \in L^{q}, q>1 /(p-1)$. All arguments up to, and including, (3.4) follow straightforwardly. Moreover, (3.4) gives

$$
\begin{aligned}
0 \leq f(x)-g(x) & \leq C(2 x(f(x)-g(x)))^{p-1} \max \left\{\mu\left(U_{\alpha}(x)\right), \mu\left(A_{\beta}(x)\right)\right\} \\
& \leq C(x(f(x)-g(x)))^{p-1}\left(\mu\left(U_{\alpha}(x)\right)+\mu\left(A_{\beta}(x)\right)\right) \\
& \leq C(x(f(x)-g(x)))^{p-1}\left(\operatorname{Area}\left(U_{\alpha}(x)\right)^{1 / q^{\prime}}+\operatorname{Area}\left(A_{\beta}(x)\right)^{1 / q^{\prime}}\right)\|\rho\|_{L^{q}} \\
& \stackrel{(3.5)}{\leq} C(x(f(x)-g(x)))^{p-1}\left(x^{2}(f(x)-g(x))\right)^{1 / q^{\prime}} \\
& =C x^{p-1-2 / q^{\prime}}(f(x)-g(x))^{p-1+1 / q^{\prime}} .
\end{aligned}
$$

The third line is due to Hölder inequality, with $q^{\prime}$ denoting the Hölder conjugate of $q$. Here $C$ may denote different (positive) constants. Conditions $p<2$ and $q>1 /(p-1)$ imply $p-1+1 / q^{\prime}>1$, which forces $f(x)=g(x)=0$ for sufficiently small $x$, hence the two curves coincide. Contradiction.

\section{Appendix. Curvature of Minimizers}

In $[19,23]$ we studied the average-distance problem considered over the family of connected 1-dimensional sets. Here we study the problem among parameterized curves. The conditions for stationarity and regularity estimates of $[19,23]$ still apply in this setting. Here we state the estimates for general $p \geq 1$, while we previously considered only $p=1$. The extension is straightforward. 
We start by stating the conditions for the case that $\mu$ is a discrete measure, $\mu=\sum_{i=1}^{n} m_{i} \delta_{x_{i}}$ where $m_{i}>0$ for all $i$ and $\sum_{i=1}^{n} m_{i}=1$. Arguing as in (Lem. 7 of [23]) we conclude that any minimizer of Problem 1.2 is a solution of a euclidean traveling salesman (for Problem 1.1 the minimizers were Steiner trees) and is thus a piecewise linear curve with no self-intersections (i.e. $\gamma$ is injective). Such $\gamma$ can be described as a graph as follows. Let $V$, the set of vertices, be the collection of all minimizers over $\Gamma_{\gamma}$ of distance to each of the point in $X=\left\{x_{1}, \ldots, x_{n}\right\}$. That is let

$$
V=\bigcup_{i=1}^{n} \operatorname{argmin}_{z \in \Gamma_{\gamma}}\left|z-x_{i}\right| .
$$

We can write $V=\left\{v_{1}, \ldots, v_{m}\right\}$ where $v$ are ordered as they appear along $\Gamma_{\gamma}$ (in increasing order with respect to parameter of $\gamma$ ). Then $\Gamma_{\gamma}$ is the piecewise linear curve $\left[v_{1}, \ldots, v_{m}\right]$.

For $j=1, \ldots, m$ let $I_{j}$ be the set of indices of points in $X$ for which $v_{j}$ is the closest point in $V$

$$
\begin{aligned}
I_{j} & =\left\{i \in\{1, \ldots, n\}:(\forall k=1, \ldots, m) \quad d\left(x_{i}, v_{j}\right) \leq d\left(x_{i}, v_{k}\right)\right\} \\
& =\left\{i \in\{1, \ldots, n\}:\left(\forall y \in \Gamma_{\gamma}\right) \quad d\left(x_{i}, v_{j}\right) \leq d\left(x_{i}, y\right)\right\} .
\end{aligned}
$$

If $i \in I_{j}$ then we say that $x_{i}$ talks to $v_{j}$. We say that a vertex $v_{j}$ is tied down if for some $i, v_{j}=x_{i}$. We then say that $v_{j}$ is tied to $x_{i}$. A vertex which is not tied down is called free. As shown in [23], if $x_{i}$ talks to $v_{j}$ and $v_{j}$ is free then $x_{i}$ cannot talk to any other vertex.

As in [23] we consider the optimal transportation plan between $\mu$ and its projection onto $\Gamma_{\gamma}$. That is, consider an $n$ by $m$ matrix $T$ such that

$$
T_{i j} \geq 0, \sum_{j=1}^{m} T_{i j}=m_{i}, \text { and } T_{i j}>0 \text { implies } i \in I_{j}
$$

Note that $\mu=\sum_{i=1}^{n} \sum_{j=1}^{m} T_{i j} \delta_{x_{i}}$. Furthermore observe that if $v_{j}$ is tied to $x_{i}$ then $i \in I_{j}$ and $T_{i j}=m_{i}$. Let $\pi=\sum_{i, j} m_{i} \delta_{x_{1}} \otimes \delta_{v_{j}}$. We note that the first marginal of $\pi$ is $\mu$ and that it describes an optimal transportation plan between $\mu$ and a measure supported on $V \subset \Gamma_{\gamma}$. We define $\sigma$ to be the second marginal of $\pi$ as before (above Lem. 2.2). Then $\sigma$ is a projection of $\mu$ onto the set $\Gamma_{\gamma}$ in that the mass of $\mu$ is transported to a closest point on $\Gamma_{\gamma}$. More precisely

$$
\sigma=\sum_{j=1}^{m} \sum_{i=1}^{n} T_{i j} \delta_{v_{j}}
$$

We note that the matrix $T$ describes an optimal transportation plan between $\mu$ and $\sigma$ with respect to any of the transportation $\operatorname{costs} c(x, y)=|x-y|^{q}$, for $q \geq 1$.

We note that in this discrete setting

$$
\begin{aligned}
E_{\mu}^{\lambda, p}(\gamma) & =\sum_{i=1}^{n} m_{i} d^{p}\left(x_{i}, \Gamma_{\gamma}\right)+\lambda \sum_{i=1}^{m-1}\left|v_{i+1}-v_{i}\right| \\
& =\sum_{j=1}^{m} \sum_{i \in I_{j}} T_{i j}\left|x_{i}-v_{j}\right|^{p}+\lambda \sum_{i=1}^{m-1}\left|v_{i+1}-v_{i}\right| .
\end{aligned}
$$

Taking the first variation in $v_{j}$ provides an extension to $p>1$ of conditions for stationarity of Lemma 9 in [23]. 
Lemma A.1. Assume that $\gamma$ minimizes $E_{\mu}^{\lambda, p}$ for discrete $\mu=\sum_{i=1}^{n} m_{i} \delta_{x_{i}}$. Let $V$ be the set of vertices as defined in (A.1) and $T$ be any matrix (transportation plan) satisfying (A.3). Then

- For endpoints $j=1$ and $j=m$ let $w=v_{2}$ if $j=1$ and $w=v_{m-1}$ otherwise.

If $p>1$ or $v_{j}$ is free then

$$
\sum_{i \in I_{j}} p T_{i j}\left(x_{i}-v_{j}\right)\left|x_{i}-v_{j}\right|^{p-2}+\lambda \frac{w-v_{j}}{\left|w-v_{j}\right|}=0 .
$$

If $v_{j}$ is tied to $x_{k}$ and $p=1$ then

$$
\left|\sum_{i \in I_{j}, i \neq k} T_{i j} \frac{x_{i}-v_{j}}{\left|x_{i}-v_{j}\right|}+\lambda \frac{w-v_{j}}{\left|w-v_{j}\right|}\right| \leq m_{k} .
$$

- If $j=2, \ldots, m-1$ then if $p>1$ or $v_{j}$ is free

$$
\sum_{i \in I_{j}} p T_{i j}\left(x_{i}-v_{j}\right)\left|x_{i}-v_{j}\right|^{p-2}+\lambda\left(\frac{v_{j-1}-v_{j}}{\left|v_{j-1}-v_{j}\right|}+\frac{v_{j+1}-v_{j}}{\left|v_{j+1}-v_{j}\right|}\right)=0 .
$$

If $v_{j}$ is tied to $x_{k}$ and $p=1$ then

$$
\left|\sum_{i \in I_{j}, i \neq k} T_{i j} \frac{x_{i}-v_{j}}{\left|x_{i}-v_{j}\right|}+\lambda\left(\frac{v_{j-1}-v_{j}}{\left|v_{j-1}-v_{j}\right|}+\frac{v_{j+1}-v_{j}}{\left|v_{j+1}-v_{j}\right|}\right)\right| \leq m_{k} .
$$

The proof of the lemma is analogous to the one in [23].

Note that the condition at a corner provides an upper bound on the turning angle in terms of the $p-1$-st moment of the mass that talks to the corner. These conditions can be used as in [19] to obtain estimates on the curvature (in the sense of a measure) of minimizers $\gamma$ of $E_{\mu}^{\lambda, p}$ for general compactly supported measures $\mu$. In particular adapting the proof of Theorem 5.1 and Lemma 5.2 of [19] implies:

Proposition A.2. Let $\mu \in \mathcal{M}$ and let $\lambda>0$ and $p \geq 1$. If $\gamma:[0, L] \rightarrow \mathbb{R}^{d}$ is an arc-length-parameterized minimizer of $E_{\mu}^{\lambda, p}$ then $\gamma^{\prime} \in B V\left([0, L], \mathbb{R}^{d}\right)$ and

$$
\left\|\gamma^{\prime}\right\|_{T V([0, L])} \leq \frac{p}{\lambda} \operatorname{diam}(\operatorname{supp}(\mu))^{p-1} \mu\left(\mathbb{R}^{d}\right) .
$$

Remark A.3. A particular consequence of this estimate is that for all $T \in[0, L), v=\lim _{t \rightarrow T+} \gamma^{\prime}(t)$ exists (see Cor. 2.23 of [18]). Furthermore since $\left|\gamma^{\prime}(t)\right|=1$ a.e. it follows that $|v|=1$. It is then straightforward to prove, using the definition of a one-sided derivative, that $\gamma$ has a right derivative $\gamma^{\prime}(T+)$ and that $\gamma^{\prime}(T+)=v$. Analogous statements hold for the left derivative.

We note that the estimate holds if we consider the same problem on the class of curves with fixed endpoints: $\gamma:[0, L] \rightarrow \mathbb{R}^{d}$ with $\gamma(0)=P$ and $\gamma(1)=Q$ with $P, Q \in \operatorname{Conv}(\mu)$. The proof is essentially the same.

A consequence of this observation is that we can formulate a localized version of the estimate. In particular let $\gamma$ be the minimizer of $E_{\mu}^{\lambda, p}$ as in the Proposition A.2. Let $\pi$ and $\sigma$ be as defined above Lemma 2.2. For any interval $I=(t, t+\delta) \subset[0, L]$

$$
\left\|\gamma^{\prime}\right\|_{T V(I)} \leq p \operatorname{diam}(\operatorname{supp}(\mu))^{p-1} \frac{1}{\lambda} \sigma(\gamma(I)) .
$$

The estimate bounds how much can the curve $\gamma$ turn within interval $I$ based on the $p-1$-st moment of the mass in $\mu$ that projects onto the set $\gamma(I)$. Let $\mu_{I}$ be the measure defined as $\mu_{I}(U)=\pi(U \times \gamma(I))$, that is the 


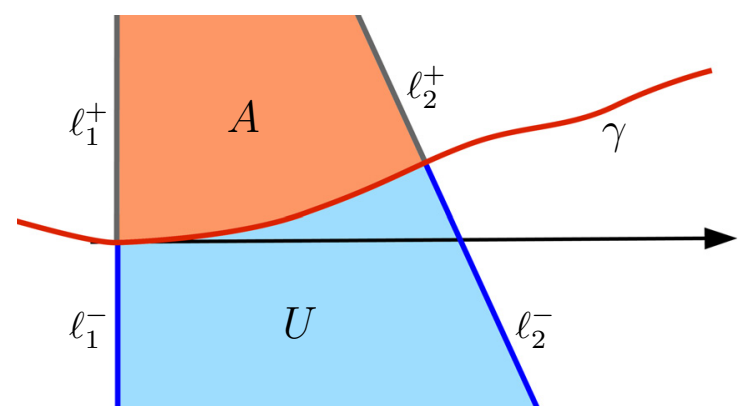

FIGURE 3. The geometry and regions relevant to obtaining one-sided turning-angle estimates.

$\mu$ measure of the set of points that projects onto $\gamma(I)$. The estimate follows from Proposition A.2 using the observation that $\left.\gamma\right|_{I}$ is a minimizer of $E_{\mu_{I}}^{\lambda, p}$ among curves which start at $\gamma(t)$ and end at $\gamma(t+\delta)$. We recall that a previous estimate on the oscillation of the tangent direction has been obtained in Theorem 3 of [17].

In this work we need finer information. We focus on dimension $d=2$. We need information not only on how much a curve turns but also on about the direction it turns in.

Lemma A.4. Consider dimension $d=2$. Let $\mu \in \mathcal{M}, \lambda>0$ and $p \geq 1$. Let $\gamma:[0, L] \rightarrow \mathbb{R}^{2}$ be an arc-lengthparameterized minimizer of $E_{\mu}^{\lambda, p}$. Let $t \in[0, L)$. By rotation and translation we can assume that $\gamma(t)=0$, $\gamma^{\prime}(t+)=e_{1}$. Let $I=(t, t+\delta)$ be such that $t+\delta<L,\left\|\gamma^{\prime}\right\|_{T V(I)}<\frac{1}{2}$. We define the region underneath the curve segment $\gamma(I)$ to be as depicted in Figure 3 . That is let $\ell_{1}^{-}=\{s(0,1): s \leq 0\}$ and $\ell_{2}^{-}=\left\{\gamma(t+\delta)+s\left(\gamma^{\prime}((t+\delta)-)\right)^{\perp}\right.$ : $s \leq 0\}$. Let $U$ be the connected component of $\mathbb{R}^{2} \backslash\left(\ell_{1}^{-} \cup \ell_{2}^{-} \cup \gamma(I)\right)$ which contains the point $\gamma\left(t+\frac{\delta}{2}\right)-\frac{\delta}{4}(0,1)$.

Let $D$ be the maximal distance of a point in $\operatorname{supp}(\mu) \cap U$, which talks to $\gamma(I)$. That is let $D=\sup \{d(x, \gamma(I))$ : $\left.x \in \operatorname{supp}(\mu) \cap U, \operatorname{argmin}_{z \in \Gamma_{\gamma}} d(x, z) \cap \gamma(I) \neq \emptyset\right\}$. Then

$$
\sup _{s \in I} \gamma^{\prime}(s) \cdot e_{2} \leq \frac{p}{\lambda} D^{p-1} \mu(U) .
$$

Proof. By approximating as in the proof of Theorem 5.1 in [19] the problem can be reduced to considering discrete measures. Thus we assume that $\mu=\sum_{i=1}^{n} m_{i} \delta_{x_{i}}$.

Let $A$ be the region above the curve segment $\gamma(I)$. That is let $\ell_{1}^{+}=\{s(0,1): s \geq 0\}$ and $\ell_{2}^{+}=\{\gamma(t+\delta)+$ $\left.s\left(\gamma^{\prime}\right)^{\perp}(t+\delta-): s \geq 0\right\}$ and let $A$ be the connected component of $\mathbb{R}^{2} \backslash\left(\ell_{1}^{+} \cup \ell_{2}^{+} \cup \gamma(I)\right)$ which contains the point $\gamma\left(t+\frac{\delta}{2}\right)+\frac{\delta}{4}(0,1)$. Note that all of the mass of $\mu$ that talks to $\gamma(I)$ is contained in $U \cup A \cup \gamma(I)$.

Due to an assumption on $I$, for all $v_{j} \in \gamma(I)$ the angle between $v_{j+1}-v_{j}$ and $e_{1}$ is less than $\pi / 4$ and so is the angle $v_{j}-v_{j-1}$ and $e_{1}$. Therefore if $i \in I_{j}$ and $x_{i} \in A$ then the directed angle between $e_{1}$ and $x_{i}-v_{j}$ is between $\pi / 4$ and $3 \pi / 4$. Therefore $\left(v_{j}-x_{i}\right) \cdot e_{2}<0$.

Let us first consider the case that $p>1$. Then from (A.8) follows that for $j$ such that $v_{j} \in \gamma(I)$

$$
\begin{aligned}
\sum_{i \in I_{j}, x_{i} \in U} p T_{i j}\left|x_{i}-v_{j}\right|^{p-2}\left(v_{j}-x_{i}\right) \cdot e_{2} & \geq \sum_{i \in I_{j}} p T_{i j}\left|x_{i}-v_{j}\right|^{p-2}\left(v_{j}-x_{i}\right) \cdot e_{2} \\
& =\lambda\left(\frac{v_{j+1}-v_{j}}{\left|v_{j+1}-v_{j}\right|}-\frac{v_{j}-v_{j-1}}{\left|v_{j}-v_{j-1}\right|}\right) \cdot e_{2} .
\end{aligned}
$$

Consider $s \in(t, t+\delta)$. Summing up over all $j$ such that $v_{j} \in \gamma((t, s))$ gives

$$
p \mu(U) D^{p-1} \geq \lambda \gamma^{\prime}(s-) \cdot e_{2},
$$

which establishes the desired claim. 
Consider now $p=1$. From (A.9) follows that for $j$ such that $v_{j} \in \gamma(I)$

$$
\begin{aligned}
\sum_{i \in I_{j}, x_{i} \in U} T_{i j} \frac{1}{\left|x_{i}-v_{j}\right|}\left(v_{j}-x_{i}\right) \cdot e_{2} & \geq \sum_{i \in I_{j}} T_{i j} \frac{1}{\left|x_{i}-v_{j}\right|}\left(v_{j}-x_{i}\right) \cdot e_{2} \\
& \geq \lambda\left(\frac{v_{j+1}-v_{j}}{\left|v_{j+1}-v_{j}\right|}-\frac{v_{j}-v_{j-1}}{\left|v_{j}-v_{j-1}\right|}\right) \cdot e_{2}-\mu\left(v_{j}\right) .
\end{aligned}
$$

Summing over $j$ such that $v_{j} \in \gamma((t, s))$ again provides the desired claim.

Acknowledgements. The authors thank the anonymous referee for valuable comments and suggestions. Both authors are thankful to FCT (grant UTA_CMU/MAT/0007/2009). XYL acknowledges the support by ICTI. XYL also thanks Universidade Nove de Lisboa for its hospitality. DS is grateful to NSF (Grant DMS-1211760) for its support. The authors would like to thank the Center for Nonlinear Analysis of the Carnegie Mellon University for its support. This research was partly carried out when XYL was affiliated with Instituto Superior Técnico.

\section{REFERENCES}

[1] H. Brézis, Functional Analysis, Sobolev Spaces and Partial Differential Equations. Universitext, Springer (2011).

[2] G. Buttazzo, A. Pratelli and E. Stepanov, Optimal pricing policies for public transportation networks. SIAM J. Optimiz. 16 (2006) 826-853.

[3] G. Buttazzo and E. Stepanov, Optimal transportation networks as free Dirichlet regions for the Monge-Kantorovich problem. Ann. Sc. Norm. Sup. Pisa Cl. Sci. 2 (2003) 631-678.

[4] G. Buttazzo and E. Stepanov, Minimization Problems for Average Distance Functionals. In vol. 14 of Calculus of Variations; Topics from the Mathematical Heritage of Ennio De Giorgi, edited by D. Pallara. Quaderni di Matematica, Caserta (2004) 47-83.

[5] G. Buttazzo and F. Santambrogio, A Model for the Optimal Planning of an Urban Area. SIAM J. Math. Anal. 37 (2005) $514-530$.

[6] G. Buttazzo and F. Santambrogio, A Mass Transportation Model for the Optimal Planning of an Urban Region. SIAM Rev. 51 (2009) 593-610.

[7] G. Buttazzo, E. Oudet and E. Stepanov, Optimal transportation problems with free Dirichlet regions. Prog. Nonlinear Differ. Eq. Appl. 51 (2002) 41-65.

[8] G. Buttazzo, E. Mainini and E. Stepanov, Stationary configurations for the average distance functional and related problems. Control Cybernet. 38 (2009) 1107-1130.

[9] G. Buttazzo, A. Pratelli, S. Solimini and E. Stepanov, Optimal Urban Networks Via Mass Transportation. Springer Lect. Notes Math. (2009).

[10] T. Duchamp and W. Stuetzle, Geometric Properties of Principal Curves in the Plane, in vol. 109 of Robust Statistics, Data Analysis, and Computer Intensive Methods, edited by H. Rieder. Springer-Verlag, Berlin (1995) 135-152.

[11] E.N. Gilbert and H.O. Pollack, Steiner minimal trees. SIAM J. Appl. Math. 12 (1968) 1-29.

[12] T. Hastie, Principal curves and surfaces. Ph.D. thesis, Stanford University (1984).

[13] T. Hastie and W. Stuetzle, Principal curves. J. Amer. Statist. Assoc. 84 (1989) 502-516.

[14] F.K. Hwang, D.S. Richards and P. Winter, The Steiner tree problem in Ann. Discrete Math. North-Holland Publishing Co., Amsterdam (1992).

[15] B. Kégl, A. Krzyzak, T. Linder, and K. Zeger, Learning and design of principal curves. IEEE Trans. Pattern Anal. Mach. Intell. 22 (2000) 281-297.

[16] A. Lemenant, A presentation of the average distance minimizing problem. Zap. Nauchn. Sem. S.-Petersburg. Otdel. Mat. Inst. Steklov. POMI 390 (2011). Translation in: J. Math. Sci. 181 (2012) 820-836.

[17] A. Lemenant, About the regularity of average distance minimizers in $\mathbb{R}^{2}$. J. Convex Anal. 18 (2011) 949-981.

[18] G. Leoni, A first course in Sobolev spaces. Grad. Stud. Math. AMS, Providence (2009).

[19] X.Y. Lu and D. Slepčev, Properties of minimizers of average-distance problem via discrete approximation of measures. SIAM J. Math. Anal. 45 (2013) 3114-3131.

[20] C. Mantegazza and A. Mennucci, Hamilton-Jacobi equations and distance functions in Riemannian manifolds. Appl. Math. Optim. 47 (2003) 1-25.

[21] E. Paolini and E. Stepanov, Qualitative properties of maximum and average distance minimizers in $\mathbb{R}^{n}$. J. Math. Sci. 122 (2004) 3290-3309.

[22] F. Santambrogio and P. Tilli, Blow-up of optimal sets in the irrigation problem. J. Geom. Anal. 15 (2005) 343-362.

[23] D. Slepčev, Counterexample to regularity in average-distance problem. Ann. Inst. Henri Poincaré (C) 31 (2014) 169-184.

[24] A.J. Smola, S. Mika, B. Schölkopf and R.C. Williamson, Regularized principal manifolds. J. Mach. Learn. 1 (2001) 179-209.

[25] R. Tibshirani, Principal curves revisited. Stat. Comput. 2 (1992) 183-190. 